\title{
Corrosion effect on the flexural strength \& micro-hardness of ips e-max ceramics
}

\author{
Cherif Mohsen \\ Fixed Prosthodontics Department, Faculty of Dentistry, Minia University, Minia, Egypt \\ E-mail: cherif.mohsen@gmail.com
}

Received 1 May 2011; revised 3 June 2011, accepted 17 June 2011.

\begin{abstract}
Objectives: The effect of ceramics construction (pressable, machinable) and corrosion on flexural strength and micro-hardness was studied. Materials \& Methods: Two types of ceramics were tested: IPS e-max Press and IPS e-max CAD. Forty samples were constructed and divided into 2 groups according to the type of ceramics. Each group was then subdivided into 2 subgroups. Subgroups 1 were not subjected to corrosion while subgroups 2 were subjected to corrosion test. Finally each subgroup was divided into 2 classes according to the type of test: biaxial flexural strength, micro-hardness. Results: There was a significant difference between the two tested ceramics as regard weight loss as IPS e-max CAD recorded less weight loss than IPS e-max Press. As regard the flexural strength, IPS e-max CAD recorded significant higher strength than IPS e-max Press. Corroded samples recorded significant lower flexural strength than non-corroded samples for the two tested ceramics. As regard the Vickers micro-hardness test, the results showed significant difference between the two tested ceramics. IPS e-max CAD recorded higher micro-hardness values than IPS e-max Press. The results also showed that the corroded samples recorded no significant micro-hardness values than non- corroded samples for the two tested ceramics. Conclusions: IPS e-max CAD recorded less weight loss weight loss after being subjected to corrosion test than IPS e-max Press. The method of fabrication affected the flexural strength \&micro-hardness of ceramic as machinable ceramic (e-max CAD) recorded significant higher data than pressable ceramic (e-mas Press). Corrosion decreased the flexural strength of both tested ceramics but had no effect on microhardness.
\end{abstract}

Keywords: IPS E-Max; Corrosion; Flexural Strength; Micro-Hardness

\section{INTRODUCTION}

Advanced progress in technology and research of new dental materials has resulted in an increased number of all-ceramic systems. Several processing techniques are available for fabricating all-ceramic restoration: sintering, heat pressing, infiltration, casting and machining. $[1,2]$ Recently, IPS e-max is an innovative all-ceramic system which covers the entire all-ceramics indication range from thin veneers to 10 units FPDs. IPS e-max delivers high strength and high esthetic materials for the press and the CAD/CAM technologies [3].

IPs e-max Press (Ivoclar Vivadent) consists of a lithium-disilicate pressed glass ceramic, but its physical properties and translucency are improved through different firing processes compared to IPs Empress 2. Emax press is a pressed glass-ceramic ingot (lithium disilicate crystals). The lithium disilicate crystals prevent the propagation of microcracks and contribute to the esthetic translucency of the Ips e.max press restorations. [4].

IPs e.max CAD is a lithium disilicate glass-ceramic block for the CAD/CAM technique. It is fabricated using an innovative process which provides an impressive homogeneity of the material. The block can be processed very easily in a CAD/CAM unit in this crystalline intermediate stage. The typical and striking color of IPs e. max CAD ranges from whitish to blue and bluish-grey microstructure of the glass-ceramic. IPs e.max CAD combines uniqueness and high performance. The innovative lithium disilicate ceramic fulfills the highest esthetic demands and unites state of the art technology with exceptional user-friendliness [5].

The CEREC in-Lab system is an evolution from the dentist-based CERECIII system. CEREC in-Lab is based on the same technology as the chairside system, with the addition of laser measurement technology. The system is a self-contain scanning and milling unit designed to fabricate single copings and three-unit FPD frameworks. The die to be scanned is placed in the system and is op- 
tically scanned. A virtual die is then displayed on the monitor and the coping is designed through the software. The die is then replaced with a block of the desired material and the coping or framework is machined. [6]

The chemical durability of ceramic materials is basically good, but it may be influenced by many factors, such as the composition and microstructure of the ceramic material, the chemical character of the corrosive medium, the exposure time, and the temperature. [7] The multiphase microstructure of many dental ceramic materials results in complicated corrosion modes, as each phase is likely to react individually to the corrosive medium. In a previous study, Midelling et al. (1999) [8] showed that different dental ceramic materials did not respond uniformly to a specific corrosive environment. In partially crystallized ceramics, immersion in $4 \%$ acetic acid at $80^{\circ} \mathrm{C}$ for $18 \mathrm{~h}$ resulted in increased surface roughness, whereas microcrystalline and non-crystalline materials remained virtually unaffected, as judged by the surface roughness test results.

Flexural strength of the ceramic material is an important factor for the success of any fixed restorations [9]. Flexural strength test are preferred to diametral compressive test because they more closely simulate the stress distribution in the dental prostheses. It was stated by Anusavice (1996) [10] that: "This test is, in a sense a collective measurement of tensile, compressive and shear stresses simultaneously." Marx \& Fisher (2001) [11] and Albakry et al. (2003) [12] compared the flexural strength of IPS e-max, IPS Empress 2 and IPS Empress. They reported that IPS e-max recorded higher flexural strength than the other 2 pressable ceramics.

Hardness is considered an important property when comparing restorative materials. It is a measure of the resistance to permanent surface indentation or penetration. The significance of measuring hardness in dental material is that it delineates the abrasiveness of a material to which the natural dentition may be submitted [13]. Albakry et al. (2003) [13] reported no difference in micro-hardness between 2 pressable ceramics: IPS Empress 2 and an experimental ceramic.

The aim of this research is to compare between IPS e-max Press \& IPS e-max CAD as regard weight loss due to a corrosive medium as well as the effect of corrosion on their flexural strength \& micro-hardness.

\section{MATERIALS \& METHODS}

\subsection{Samples Grouping}

Forty samples were constructed and divided into 2 groups (20 samples each) according to the type of ceramics: IPS e-max Press \& IPS e-max CAD .Each group was then subdivided into 2 subgroups(10 samples each). Subgroups 1 were not subjected to corrosion while sub- groups 2 were subjected to corrosion test. Finally each subgroup was divided into 2 classes ( 5 samples each) according to the type of test: biaxial flexural strength, micro-hardness.

\subsection{Samples Construction}

\subsubsection{IPS E-Max Press}

A cylindrical split copper mold was designed and constructed to allow the fabrication of wax discs with the intended dimension $(10 \mathrm{~mm}$ diameter and $1.5 \mathrm{~mm}$ thickness). The wax was then molten and poured into the copper mold. After the wax hardened, it was smoothened with a nylon cloth, sprued. The wax were invested according to the IPs press vest speed investment instructions for use. The corresponding IPs silicone ring then carefully filled with the investment material up to the mark and the ring gauge was placed with a hinged movement. The investment material was allowed to set without manipulating the investment ring. Then the investment ring was prepared for preheating as follows: ring gauge and ring base were removed and the investment ring was pushed out of the IPs silicone ring. Rough spots on the bottom surface of the investment ring were removed with a plaster knife to insure flat smooth base. The investment ring with the opening facing down was placed in the preheating furnace and heated at $850^{\circ} \mathrm{C}$ for 60 minutes. Once the preheating cycle had been completed, the investment ring was removed from the preheating furnace, the cold IPs e.max press ingot was inserted into the hot investment ring. The powder-coated cold IPs e.max Alox plunger was placed into the hot investment ring.

The completed investment ring was placed at the center of the hot press furnace (EP600 Combi) using the investment tongs and start button was pressed to start the selected program. The investment ring was removed from the furnace immediately after the program was completed and placed on the cooling grid and allowed to cool to room temperature. Then, divesting was performed and the reaction layer formed during the press procedure was removed using IPS e-max Invex liquid followed by air blasting. Subsequently, the ceramic samples were cleaned under running water and then air dried. Sprue was then cut using a fine diamond disk (916-200) and the samples were finished by air blasted with $\mathrm{Al} 2 \mathrm{O} 3$ at 1 bar, and cleaned with steam.

Finally, the samples were subjected to a glaze firing cycles using IPS e-max ceram glaze paste according to manufactures' instructions.

\subsubsection{IPS E-Max CAD}

An extra hard type four stone material "Dentona" which is recommended for $\mathrm{CAD} / \mathrm{CAM}$ models, was vacuum mixed with the recommended water/powder ratio, vi- 
brated and poured inside the copper mold with the intended dimension $(10 \mathrm{~mm}$. diameter and $1.5 \mathrm{~mm}$ thickness), which is placed on a clean and dry glass slab. Another glass slab was adjusted on the upper surface of the mold in order to obtain smooth and flat surface. After complete setting of the stone model, the mold was splitted, the model was trimmed and ready for scanning. The disc model was mounted on the model holder using a special filling material recommended by the manufacturer to fix the model in place while scanned. The scanned images were stored on the computer hard disc ready for the sample design step. Following scanning, the sample was designed and milled using IPS e-max CAD block. Finally, the samples were subjected to crystallization \& glaze firing with IPS e-max CAD Crystal/ glaze paste. The IPs e.max CAD samples attained their final physical properties, and also desired aesthetic properties, such as color, excellent translucency and brightness.

\subsubsection{Corrosion Test}

The samples were first washed three times with ethyl alcohol then dried. The samples were weighed then placed in test tubes having the same volume $4 \%$ acetic acid solution at a temperature of $80^{\circ} \mathrm{C}$ and kept for 16 hours according to the ISO 6872 Standards for hydrolytic resistance of dental ceramic materials [8]. To reduce the risk of micro-crack formations, the temperature of the corrosive solution was slowly increased until the storage temperature of $80 \pm 2^{\circ} \mathrm{C}$ was reached. After cooling to room temperature at the end of the test the samples were removed, rinsed with de-ionized distilled water and ethyl alcohol and dried. Then the samples were washed with water, dried and re-weighed. The weight loss was then calculated in relation to the surface area of the samples: weight of the sample before chemical degradation test minus its weight after chemical degradation test divided by the surface area of the sample [14] .

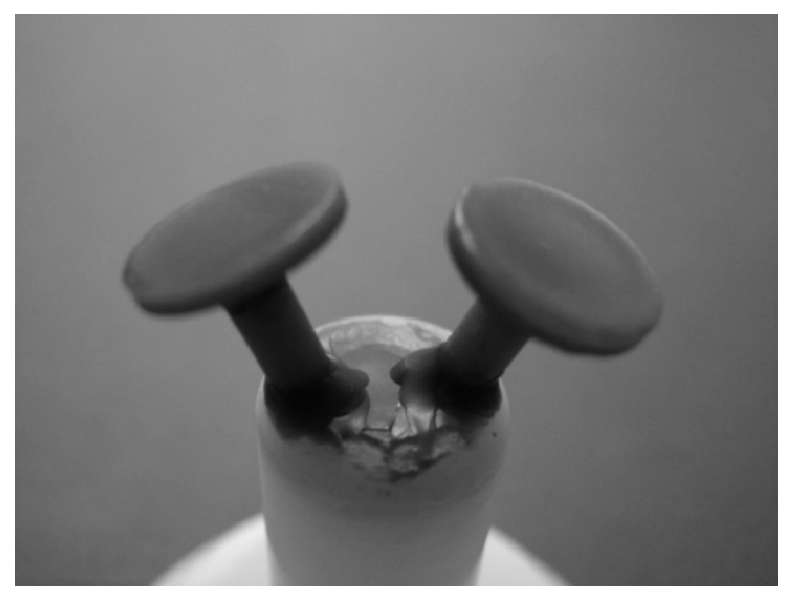

Figure 1. Sprued disc patterns attached to the ring base.

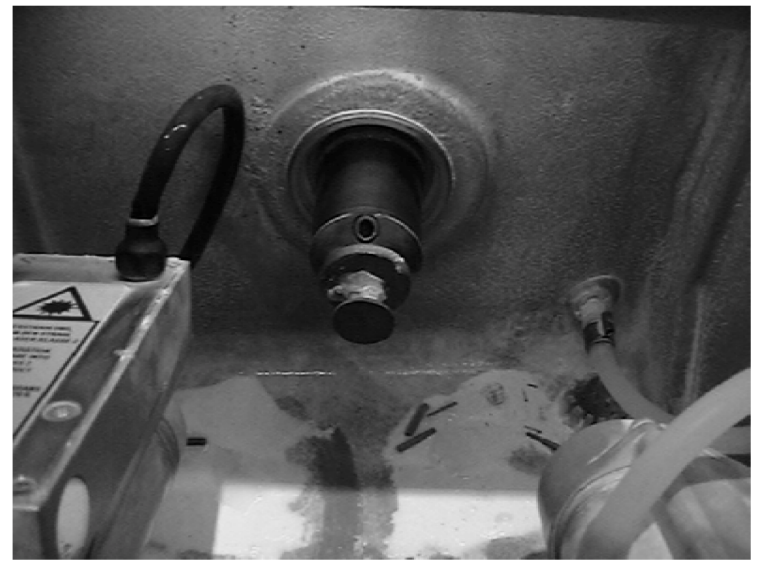

Figure 2. Disc stone model mounted on the model holder.

\subsubsection{Biaxial Flexural Strength Test}

According to the ASTM Standard (ASTM, F394-789, 1991) standard test method for flexural strength of ceramic [15] and the international standard ISO6872-1994 [16], the tested samples were fractured using the piston on a three ball technique. The test assembly was made of a copper base ring having a centralized circle with three equally distributed balls and a steel piston. The base was attached to the lower fixed part of the testing machine (Lloyd universal testing machine, Lloyd Instruments, U.K.). The discs were placed in the centralized circle and supported on the three ball bearings. The piston was attached to the under-side of the upper movable part of the testing machine. Discs were loaded to failure by a compressive load applied by the piston. A cross head loading rate of $0.5 \mathrm{~mm} /$ minute was applied .This test assembly allowed the maximum fracture force to be generated at the center of the tested disc. The flexural strength was calculated according to equations developed by Marshall [17], Wachtman [18] and Kirstein and Woolley [19] .

(1) $\sigma=A P / t^{2}$

$$
\begin{aligned}
& A=\left(3 / 4 \pi\left[2(1+v) \ln \left(a / r_{0}^{*}\right)\right.\right. \\
& \left.\left.+(1-v)\left(2 a^{2}-r_{0}^{* 2}\right) / 2 b^{2}+(1+v)\right]\right)
\end{aligned}
$$

(3) $r_{0}^{*}=\left(1.6 r_{0}^{2}+t^{2}\right)^{1 / 2}-0.675 t$

Where $: \sigma=$ failure stress

$P=$ applied load at failure

$t=$ thickness of the sample $v=$ Poisson's ratio $(0.25)$

$a=$ radius of the support circle

$b=$ radius of the tested sample

$r_{0}=$ radius of the piston in contact

\subsubsection{Hardness Test}

The surface hardness were measured using Vickers Hard- 
ness Tester (Model HVS-50, Laizhou Huayin Testing Instrument Co Ltd. China). The tested sample was mounted in the horizontal stage of the tester then the indentor was lowered under a load of $10 \mathrm{Kg}$ for $20 \mathrm{sec}-$ onds. The indentations were measured immediately after automatic return back of the indentor .

Readings were performed by measuring the size of the diagonals of the indentation directly in Vickers. For each tested sample, 5 indentations were measured and an average Vickers hardness number $H_{V}$ was determined .

\subsubsection{Statistical Test}

Data was collected, calculated, tabulated. and presented as means. The ion leached results were further statistically analyzed using one-way ANOVA Test. Then, a Tukey Test was performed to determine significant differences between the tested groups using a confidence level of $0.05(p<0.05)$.

\section{RESULTS}

\subsection{Corrosion Test Results}

Means and standard deviations of the ceramics weight loss for the tested groups are presented in Table $\mathbf{1}$ and Figure 3. A one way ANOVA Test was used to determine significant differences between the tested samples $(p<0.05)$. The Tukey test for multiple comparisons of means at $(p<0.05)$ was done following the one-way analysis of variance. The results showed a statictical significant difference between the two tested ceramics. IPS e-max CAD recorded less weight loss than IPS emax Press.

\subsection{Biaxial Flexural Strength Results}

Means and standard deviations of the ceramics flexural

Table 1. Means \& Standard deviations of weight Loss $\left(\mathrm{mg} / \mathrm{cm}^{2}\right)$ of the tested materials after the corrosion test.

\begin{tabular}{cccc}
\hline Materials & IPS e-max Press & IPS e-max CAD & $\begin{array}{c}\text { Critical Value } \\
03.4\end{array}$ \\
\cline { 1 - 3 } Weight Loss & $10.8(1.3)$ & $06.4(1.1)$ & \\
\hline
\end{tabular}

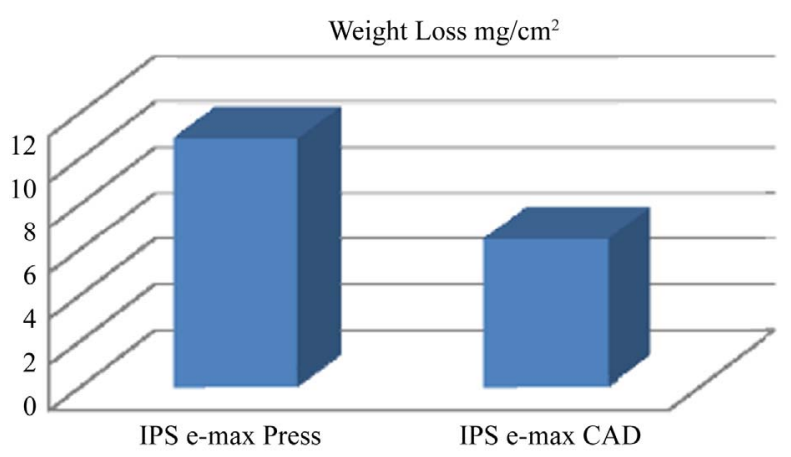

Figure 3. Comparison between the weight loss due to corrosion test of the tested ceramics. strength data recorded for the tested groups are presented in Table 2 and Figure 4. A one way ANOVA Test was used to determine significant differences between the tested samples $(p<0.05)$. The Tukey test for multiple comparisons of means at $(p<0.05)$ was done following the one-way analysis of variance .The results showed that IPS e-max CAD recorded significant higher strength than IPS e-max Press.

Corroded samples recorded significant lower flexural strength than non-corroded samples for the two tested ceramics.

\subsection{Micro-Hardness Results}

Means and standard deviations of the ceramics microhardness values for the tested groups are presented in Table 3 and Figure 5. A one way ANOVA Test was used to determine significant differences between the tested samples $(p<0.05)$. The Tukey test for multiple comparisons of means at $(p<0.05)$ was done following the one-way analysis of variance. The results showed significant difference between the two tested ceramics. IPS e-max CAD recorded higher micro-hardness values than IPS e-max Press. The results also showed that the corroded samples recorded no significant micro-hard- ness values than non-corroded samples for the two tested ceramics.

\section{DISCUSSION}

Improvement of appearance are continuously increasing, the use of all-ceramic materials became increasingly

Table 2. Means \& Standard deviations of flexural strength (MPa) of the tested ceramics before \& after corrosion test.

\begin{tabular}{cccccc}
\hline Materials & \multicolumn{2}{c}{ IPS e-max Press } & \multicolumn{2}{c}{ IPS e-max CAD } & \\
\cline { 1 - 3 } $\begin{array}{l}\text { Corrosion } \\
\text { condition }\end{array}$ & $\begin{array}{c}\text { Without } \\
\text { corrosion }\end{array}$ & $\begin{array}{c}\text { After } \\
\text { corrosion }\end{array}$ & $\begin{array}{c}\text { Without } \\
\text { corrosion }\end{array}$ & $\begin{array}{c}\text { After } \\
\text { corrosion }\end{array}$ & $\begin{array}{c}\text { Value : } \\
20\end{array}$ \\
$\begin{array}{l}\text { Flexural } \\
\text { strength }\end{array}$ & $318(12)$ & $257(12)$ & $345(7)$ & $289(8)$ & \\
\hline
\end{tabular}

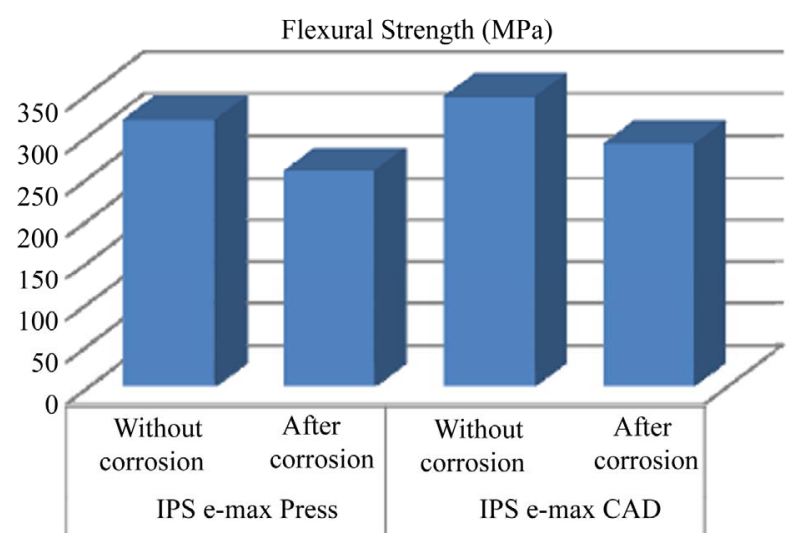

Figure 4. Comparison between the flexural strength of the tested ceramics. 
Table 3. Means \& Standard deviations of micro-hardness (HV) of the tested ceramics before $\&$ after corrosion test.

\begin{tabular}{|c|c|c|c|c|c|}
\hline Materials & \multicolumn{2}{|c|}{ IPS e-max Press } & \multicolumn{2}{|c|}{ IPS e-max CAD } & \multirow{4}{*}{$\begin{array}{c}\text { Crtitical } \\
\text { Value: } \\
37.40\end{array}$} \\
\hline $\begin{array}{l}\text { Corrosion } \\
\text { condition }\end{array}$ & $\begin{array}{l}\text { Without } \\
\text { corrosion }\end{array}$ & $\begin{array}{l}\text { After } \\
\text { corrosion }\end{array}$ & $\begin{array}{l}\text { Without } \\
\text { corrosion }\end{array}$ & $\begin{array}{l}\text { After } \\
\text { corrosion }\end{array}$ & \\
\hline $\begin{array}{l}\text { Micro- } \\
\text { hardness }\end{array}$ & $\begin{array}{l}453.25 \\
(23)\end{array}$ & $427.12(12)$ & $545.68(16)$ & $528.13(17)$ & \\
\hline $\begin{array}{c}\text { Difference } \\
\text { in Hardness } \\
(\mathrm{DH})\end{array}$ & \multicolumn{2}{|c|}{26.13} & \multicolumn{2}{|c|}{17.55} & \\
\hline
\end{tabular}

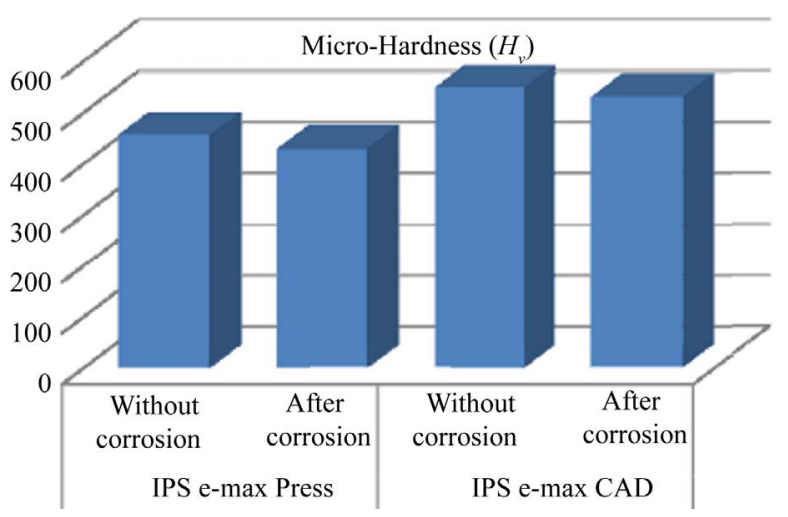

Figure 5. Comparison between the micro-hardness of the tested ceramics.

relevant to restorative dentistry. All-ceramic restorations are characterized by enhanced esthetic properties, high biocompatibility, diminished plaque accumulation, low thermal conductivity, abrasion resistance and color stability. To achieve all-ceramic restorations with appropriate fracture strength, exceeding the maximal biting force, new ceramic core materials were recently introduced. In the current study, IPs e.max press and IPs e.max CAD were selected as being recommended for use in posterior crowns because of the improved mechanical properties [20]. The $1.5 \mathrm{~mm}$ thickness used for the samples is the thickness indicated for the occlusal surface for fixed restorations $[4,5]$.

Cerec in-Lab 3D system was used as it is the latest addition to Sirona CAD/CAM product line, introduced in 2005. Its advanced software allows for broad range of indications: crown copings, multi-unit bridge frameworks, inlays, onlays and fully contoured crowns out of single solid blocks. It also allows anatomically perfect results due to the bio-generic occlusal surface design of inlays and onlays. The bio-generic modeling function is based on data acquired from thousands of natural teeth. The preparation margin is marked with just a few mouse clicks and the software does all the rest. In order to ensure the accuracy of the restoration, the lab technician sees what will be milled on the screen before it is sent to the milling machine. Milling performance and precision has been optimized to \pm 25 microns. [21].

Dental ceramics are often called inert materials [22]. Corrosion rate and behavior of ceramics are more favorable than dental alloys due to the fact that silica has the lowest known permeability to oxygen [23]. It can be hypothesized, however, that differences in the composition, microstructure and environmental conditions will affect the degree of corrosion degradation [22]. Hammad and khalil [24] reported that ceramic surfaces finished using different techniques reacted differently when subjected to etching using fluoride agents. In this study, the corrosion test used was according to the ISO 6872 Standards for hydrolytic resistance of dental ceramic materials. [8]

Strength measurements of a brittle dental material is often relied upon as an indicator of its structural performance [25]. In this research, the biaxial flexural strength was measured for the tested ceramics. The biaxial flexural tests was added to the ISO standard for dental ceramics (ISO 6872-1994) [25]. There are different designs for the biaxial tests, which include ball-onring, ring-on-ring and piston-on-three-ball test (26). The latter is the ASTM Standard (ASTM, F394-789, 1991) [1,26-27] for biaxial flexure testing and was chosen for this study. Flexural strength test are preferred to diametral compressive test because they more closely simulate the stress distribution in the dental prostheses. This test also, eliminates the effect of edge fracture because they are not directly loaded, and the maximum tensile stress occur within the central loading area [28, 29].

Several properties of a material are related to its hardness as strength, proportional limit and ductility. [10,30] Indentation hardness testing is a convenient means of investigating the mechanical properties of a small volume of materials. Among a variety of indentor geometries used in hardness testing, the Vickers indentor is one in most widespread use. The Vicker Hardness Test was selected because it is suitable for determining the hardness of small areas as used by previous investi- gator [30].

The corrosion behavior of ceramics is generally smaller compared to other dental alloys due to the fact that silica has the lowest known permeability to oxygen [23]. Garcia et al. (2003) [31] showed that veneering base metal alloys with ceramics reported lower passive current densities due to the good corrosion resistance of ceramics. In dental literature, corrosion behavior of the ceramic materials is not well documented .The concept of this research is based on the hypothesis made by Midelling et al. (2001) [22]. They drew the attention that due to variations existing in the microstructure and composition 
between dental ceramic materials, it may be hypothesized that these interceramic differences will qualitatively and quantitatively influence the ion leaching when the ceramics are exposed to aqueous media. The results reported in this research showed statistical significant difference between the two tested ceramics; IPS e-max CAD showed better chemical stability by recording less weight loss values than IPS e-max Press. This may be due to the fact of difference in the technology of fabrication as the IPS e-max CAD was subjected to 1 firing cycles (crystallization \& glaze firing) compared to the IPS e-max Press which was subjected to 2 firing cycles (pressing then glazing) as the corrosion process of a material can be affected by the applied heat treatment [32].

The results of this study revealed that IPS e.max CAD samples recorded a higher flexural strength means value (345) MPa than that obtained with IPS e.max Press samples (318) MPa before corrosion. The statistical analysis showed a significant difference between e.max CAD flexural strength mean value and that of IPS e.max Press. This can be explained as the tightly controlled industrial ceramic processing produces increased micro-structural uniformity, higher density, lower porosity and lesser residual stresses with a smaller range of fracture strength variation and therefore greater structural reliability for dental applications than laboratory- processed dental ceramic material [33]. The results also showed that for both types of tested ceramics, the corroded samples recorded significant less flexural strength than that of the non-corroded samples. This may be due to the chemical degradation of ceramics in an aqueous environment. Under more severe conditions, as in an acidic solution for chemical durability test, the $\mathrm{Si}-\mathrm{O}-\mathrm{Si}$ bonds may be broken, and the entire glass structure may be impaired. It can be assumed that similar degradation processes will also occur for many dental ceramics because their microstructure is dominated by a glassy matrix [32].

As regard micro-hardness, the results showed that the e.max CAD samples recorded a higher micro-hardness value (545.68) $H_{V}$ than that obtained with e.max Press samples (453.25) $H_{V}$ for the samples not subjected to corrosion. Although, both ceramics composition are nearly the same, the statistical difference may be due to the difference in the technology of fabrication as e-max CAD is subjected to milling then heat treatment, while it is in solid state. On the other hand, e-max Press is softened by heat in the pressing step, then subjected to a removal of the reaction layer then subjected to heat treatment. The corrosion test had insignificant effect on hardness as reported by previous investigator. [34] The results showed no statistical difference between the corroded and the non-corroded samples for both ceramics tested.

\section{CONCLUSIONS}

1- IPS e-max CAD recorded less weight loss after being subjected to corrosion test than IPS e-max Press.

2- The method of construction affected the flexural strength \& micro-hardness of ceramic as machinable ceramic (IPS e-max CAD) recorded significant higher data than pressable ceramic (IPS e-mas Press).

3- Corrosion decreased the flexural strength of both tested ceramics .

4- Corrosion test had insignificant effect on hardness.

\section{REFERENCES}

[1] Cattell, M.J., Knowles, J.C., Clarke, R.L. and Lynch, E. (1999) The biaxial flexural strength of two pressable ceramic systems. Journal of Dentistry, 27, 183-196. doi:10.1016/S0300-5712(98)00047-5

[2] Lawn, B.R., Deng, Y. and Thompson, V.P. (2001) Use of contact testing in the characterization and desing of allceramic crownlike layer structures. Journal of Prosthetic Dentistry, 86, 495-510. doi:10.1067/mpr.2001.119581

[3] Dickerson, W. and Miyasaki, M. (1999) The esthetic revolution continues-IPS Empress. Journal of Oral Health, 2, 87-90.

[4] Update technical. (2005) Ivoclar vivadent technical, manufactuer's production manual. Ivoclar Vivadent.

[5] IPS e-max CAD, Lab+to+Dentist (2005) www.ivoclarvivadent.com, Ivoclar Vivadent.

[6] McLaren, E.A. and Terry, D.A. (2002) CAD/CAM systems, materials and clinical guidelines for all-ceramic crowns and fixed partial dentures. Compendium Continuing Education Dentistry, 23, 637-641.

[7] White, W.B. (1992) Theory of corrosion of glass and ceramics. In: Clarke, D.E. and Zoitos, B.K., Eds. Corrosion of Glass, Ceramics and Superconductors. Noyes Publications, Park Ridge, 2, 2-28.

[8] Milleding, P., Wenneberg, A., Alaeddin, S. and Karlesson, S. and Simon, E. (1999) Surface corrosion of dental ceramics in vitro. Biomaterials, 26, 733-746. doi:10.1016/S0142-9612(98)00223-3

[9] Kroeze, H.J., Plasschaert, A.J., Van't Hof, M.A. and Truin, G.J. (1990) Prevalence and need or replacement of amalgam and composite restoration in Dutch adults. Journal of Dental Research, 69, 1270-1274. doi:10.1177/00220345900690060901

[10] Anusavice, K.J. (1996) Phillips Science of dental materials.10th Edition, WB Saunders Co., Philadelphia.

[11] Marx. R. and Fischer, H. (2001) Interner Bericht an Ivoclar Vivadent AG.

[12] Albakry, M., Guazzato, M. and Swain, M.V. (2003) Biaxial flexural strength, elastic moduli and $\mathrm{x}$ ray diffraction characterization on three pressable all-ceramic materials. The Journal of Prosthetic Dentistry, 89, 374-380. doi:10.1067/mpr.2003.42

[13] Albakry, M., Guazzato, M. and Swain, M.V. (2003) Fracture toughness and hardness evaluation of three pressable all-ceramic dental materials. Journal of Dentistry, 31,181-188. doi:10.1016/S0300-5712(03)00025-3

[14] Anusavice, K.J. and Zhang, N.-Z. (1997) Chemical 
durability of Dicor and Lithia-based glass-ceramics. Dental Materials, 13, 13-19. doi:10.1016/S0109-5641(97)80003-6

[15] Sadighpour, L., Geramipanah, F., Raeesi, B. (2006) In vitro mechanical tests for modern dental ceramics. Journal of Dentistry, Tehran University of Medical Sciences, Tehran, 3, 143-152.

[16] ISO. (1995) Dental ceramic ISO Standard 6672 - 1995, International organization for Standardization.

[17] Marshall, D.B. (1980) An improved biaxial flexure test for ceramics. American Ceramic Society Bulletin, 59, 551-553.

[18] Wachtman J.R. Jr, Capps, W. and Mandel, J. (1972) Biaxial flexure tests of ceramic substrates. Journal of Materials, 7, 188-194.

[19] Kirstein, A.F. and Woolley, R.M. (1967) Symmetrical bending of thin circular elastic plates of equally spaced point supports. Journal of research of the National Bureau of Standards, 71(C), 1-10.

[20] Wassermann, A., Kaiser, M. and Strub, J.R. (2006) Clinical long term results of VITA in-ceram classic crowns and fixed partial dentures: A systematic literature review. International Journal of Prosthodontics, 19, 355-363.

[21] Filser, F., Kocher, P. and Weibel, F. (2001) Reliability and strength of all-ceramic dental restorations fabricated by direct ceramic machining (DCM). International Journal of Computerized Dentistry, 4, 89-106.

[22] Midelling, P., Haraldsson, C. and Karlsson, S. (2002) Ion leaching from dental ceramics during static in vitro corrosion testing. Journal of Biomedical Materials Research, 61, 541-550. doi:10.1002/jbm.10109

[23] Jacobson, N.S., Opila, E.J. and Lee, K.N. (2001) Oxidation and corrosion of ceramics and ceramic matrix composites. Current Opinion in Solid State and Materials Scvience, 5, 301-309. doi:10.1016/S1359-0286(01)00009-2

[24] Hammad, I.A. and Khalil, A.M. (1994) The effect of fluo-ride treatments on glazed and polished ceramic surfaces. Egyptian Dental Journal, 40, 757.

[25] Kelly, J.R. (1995) Perspectives on strength. Dental Materials, 11, 103-110. doi:10.1016/0109-5641(95)80043-3

[26] Thompson, G.A. (2004) Determing the slow crack growth parameter and Weibull two-parameter estimates of bilaminate disks by constant displacement-rate flexural testing. Dental Materials, 20, 51-62. doi:10.1016/S0109-5641(03)00068-X

[27] Wagner, W.C. and Chu, T.M. (1996) Biaxial flexural strength and indentation fracture toughness of three new dental core ceramics. The Journal of Prosthetic Dentistry, 76, 140-144. doi:10.1016/S0022-3913(96)90297-8

[28] Ban, S. and Anusavice, K.J. (1990) Influence of test method on failure stress of brittle dental materials. Journal of Dental Research, 69, 1791-1799. doi:10.1177/00220345900690120201

[29] Wagner, W.C., O’Brien, W.J. and Mora, G.P. (1992) Fracturesurface analysis of a glaze strenghtened magnesia core material. International Journal of Prosthodontics, 5, 475-478.

[30] Craig, R.G. and Powers, J.M. (2002) Restorative dental materials, 11th Edition, Mosby Inc., St Louis.

[31] Garcia, C., Galliano, P. and Cerè, S. (2003) Electrochemical evaluation of resistance to localized corrosion of vitreous coatings containing particles app- lied on metallic substrates for biomedical applications. Materials Letters, 57, 1810-1814. doi:10.1016/S0167-577X(02)01073-X

[32] Grambow, B. (2000) Corrosion of glass. In: Revie, R.W., Ed., Uhlig's corrosion handbook, 2nd Edition, Wiley, New York, 411-437.

[33] Mc Laren, E.A. and White, S.N. (2000) Glass-infiltrated zirconia/alumina-based ceramic for crowns and fixed partial dentures: Clinical and laboratory guidelines. Quintenssence Dental Technology, 23, 63-76.

[34] Mohsen, S.A. (2004) Corrosion behavior and hardness of veneering materials. Egyptian Dental Journal, 50, 807. 